\title{
Editorial
}

\section{Risk Factors of Allergic Disease: A Study with a Large Data Set}

\author{
In-Hwan Oh, ${ }^{1}$ Yeong-Ho Rha, ${ }^{2}$ Takao Fujisawa, ${ }^{3}$ and Kyung Suk Lee ${ }^{4}$ \\ ${ }^{1}$ Department of Preventive Medicine, College of Medicine, Kyung Hee University, Seoul, Republic of Korea \\ ${ }^{2}$ Department of Pediatrics, School of Medicine, Kyung Hee University, Seoul, Republic of Korea \\ ${ }^{3}$ Institute for Clinical Research, Mie National Hospital, Mie, Japan \\ ${ }^{4}$ Department of Pediatrics, CHA Bundang Medical Center, CHA University School of Medicine, Seongnam, Republic of Korea
}

Correspondence should be addressed to In-Hwan Oh; ihoh@khu.ac.kr

Received 5 September 2017; Accepted 5 September 2017; Published 10 October 2017

Copyright (C) 2017 In-Hwan Oh et al. This is an open access article distributed under the Creative Commons Attribution License, which permits unrestricted use, distribution, and reproduction in any medium, provided the original work is properly cited.

Allergic diseases (e.g., asthma, allergic rhinitis, and atopic dermatitis) are a major public health problem, and the prevalence among children is increasing worldwide [1, 2]. Many studies have revealed the cause, pathogenesis, prevalence, and risk factors for allergic diseases for decades. However, we do not know all fields of allergic diseases and we have still explored the risk factors for allergic diseases.

Nowadays, large amounts of data (big data) are digitally stored and have been successfully used in many fields such as science, economics, and politics [3]. The interest and application of big data in medical science is also growing because big data have several advantages that expand the capacity to generate and disseminate new knowledge [3]. The sources of big data intended for medical application include government agencies, medical organizations, and other credible healthrelated institutions [3-5]. Regarding allergic diseases, big data is useful in investigating the causes, epidemiology, genetics, treatment, and economic burden [4-6].

In this special issue, six studies were published regarding the risk factors of allergic diseases.

W. S. Lee et al. analyzed the relationship between home remodeling, food allergy, and atopic dermatitis among children in Seongnam, Korea. In this large population study, home remodeling (odds ratio $=3.40$ ) and food allergy (odds ratio $=3.95$ ) are the risk factors of atopic dermatitis. K. S. Lee et al. also found that age, smoking, and elevated total $\operatorname{IgE}$ levels were other risk factors for atopic dermatitis in Korean children and adolescents by using the 2010 Korea National Health and Nutrition Examination Survey (KNHANES) which comprised a large sample. Y. Feng et al. investigated the prevalence and features of ocular allergy and comorbidities among school children in Shanghai, China, by using a questionnaire. They found a $28 \%$ prevalence of symptoms of ocular allergy. S.-J. Yi et al. investigated the risk for atopic eczema among children living in areas surrounded by large and busy roads in Seoul, Korea. They found that the onset of atopic eczema correlated with road density (odds ratio: 1.08) and proximity (odds ratio: 1.15) but not asthma and rhinitis. The study by S.-M. Koo et al. is the only one that focused on the adult population in this special issue. They analyzed the trend of use of asthma medication among asthmatic pregnant women using the Health Insurance Review and Assessment Service (HIRA) database of Korea. They found that despite the low adherence to asthma medication, exacerbations were less frequent during pregnancy. S. H. Choi et al. investigated the repeatability and safety of measuring lung function using impulse oscillation systems during bronchoprovocation testing of preschool children.

Most of the studies in this special issue focused on the relationship between environmental risk factors and allergic diseases in children and found or confirmed the relationship using large data sets. These results proved that finding the risk factors for allergic diseases using big data is promising.

This special issue is just the beginning of big data research in allergic diseases. We hope that readers will be interested in allergic diseases and big data and that this special issue could help them to devote their time to research.

In-Hwan Oh

Yeong-Ho Rha

Takao Fujisawa

Kyung Suk Lee 


\section{References}

[1] S. Halken, "Prevention of allergic disease in childhood: Clinical and epidemiological aspects of primary and secondary allergy prevention," Pediatric Allergy and Immunology, Supplement, vol. 15, no. 16, pp. 1-32, 2004.

[2] S. Hong, D. K. Son, W. R. Lim et al., "The prevalence of atopic dermatitis, asthma, and allergic rhinitis and the comorbidity of allergic diseases in children," Environmental Health and Toxicology, vol. 27, p. e2012006, 2012.

[3] T. B. Murdoch and A. S. Detsky, "The inevitable application of big data to health care," The Journal of the American Medical Association, vol. 309, no. 13, pp. 1351-1352, 2013.

[4] P. J. Gergen, S. J. Arbes Jr., A. Calatroni, H. E. Mitchell, and D. C. Zeldin, "Total IgE levels and asthma prevalence in the US population: Results from the National Health and Nutrition Examination Survey 2005-2006," Journal of Allergy and Clinical Immunology, vol. 124, no. 3, pp. 447-453, 2009.

[5] T. Zuberbier, J. Lötvall, S. Simoens, S. V. Subramanian, and M. $\mathrm{K}$. Church, "Economic burden of inadequate management of allergic diseases in the European Union: A GA2LEN review," Allergy: European Journal of Allergy and Clinical Immunology, vol. 69, no. 10, pp. 1275-1279, 2014.

[6] R. A. Ortiz and K. C. Barnes, "Genetics of allergic diseases," Immunology and Allergy Clinics of North America, vol. 35, no. 1, pp. 19-44, 2015. 


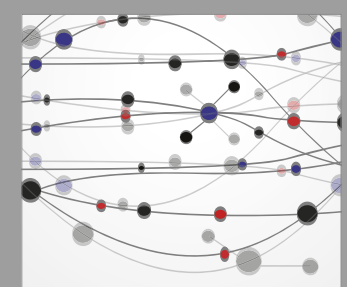

The Scientific World Journal
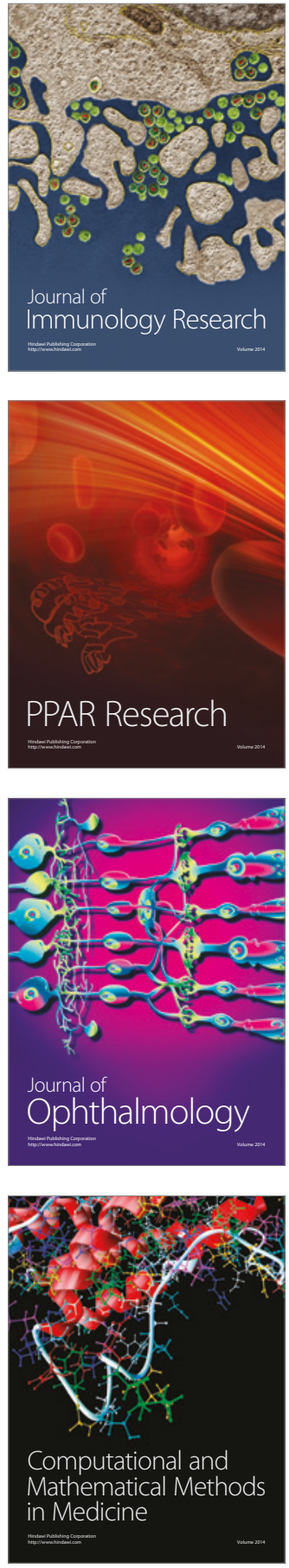

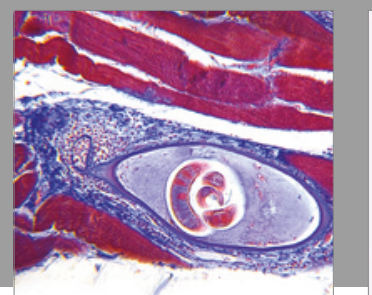

Gastroenterology Research and Practice
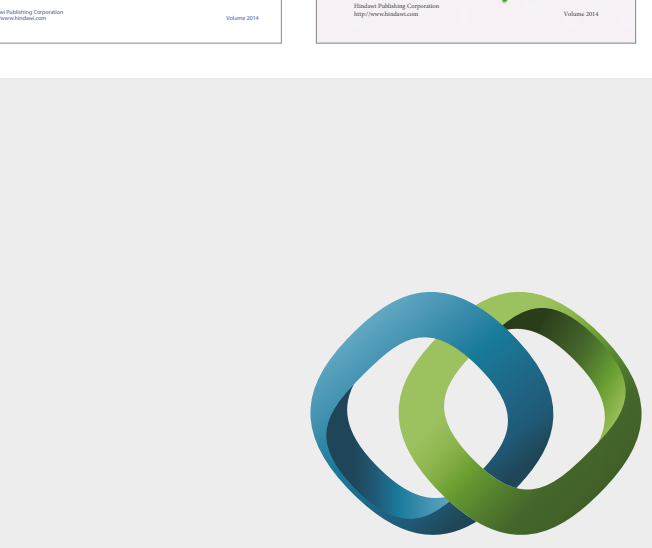

\section{Hindawi}

Submit your manuscripts at

https://www.hindawi.com
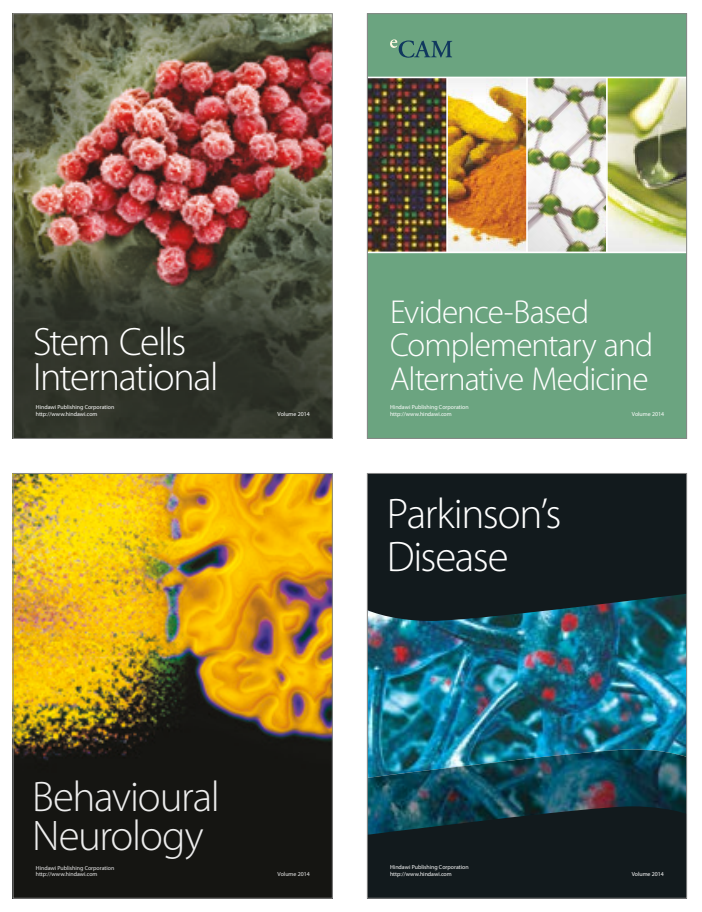
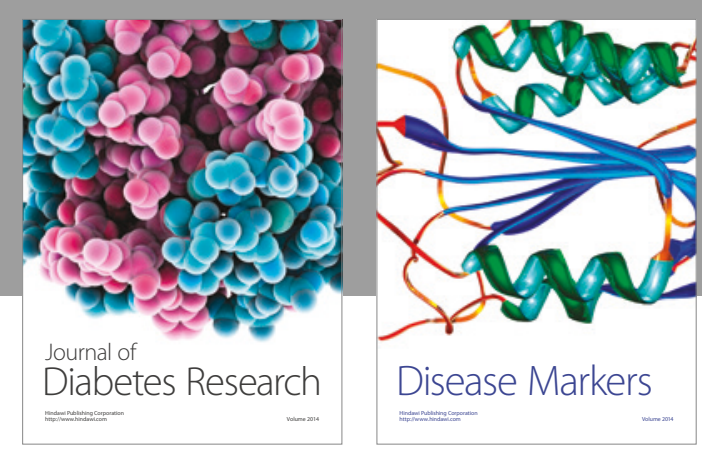

Disease Markers
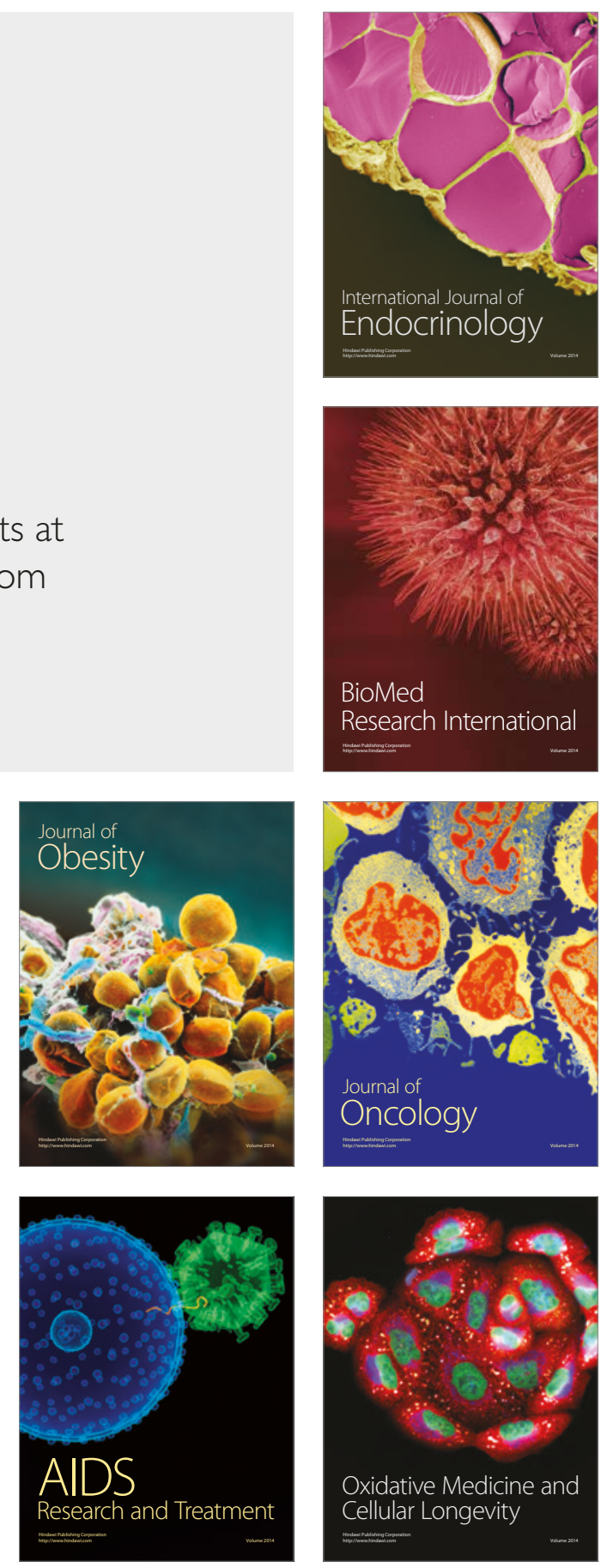